\title{
REPRESENTATIONS OF *-REGULAR RINGS AND THEIR ORTHOLATTICES OF PROJECTIONS
}

\author{
CHRISTIAN HERRMANN AND NIKLAS NIEMANN
}

\begin{abstract}
We show that a subdirectly irreducible $*$-regular ring admits a representation within some inner product space provided so does its ortholattice of projections.
\end{abstract}

\section{INTRODUCTION}

The motivating examples of $*$-regular rings, due to Murray and von Neumann, were the $*$-rings of unbounded operators affiliated with finite von Neumann algebra factors; to be subsumed, later, as $*$-rings of quotients of finite Rickart $C^{*}$-algebras. All the latter have been shown to be $*$-regular and unit-regular (Handelman [7]). Representations of these as *-rings of endomorphisms of suitable inner product spaces have been obtained first, in the von Neumann case, by Luca Giudici (cf. [19]), in general in joint work with Marina Semenova [13].

The principal right ideals of a $*$-regular ring $R$ form a modular ortholattice $\mathrm{L}^{\perp}(R)$, also to be viewed as the ortholattice of projections of $R$. As observed by Giudici [4], any representation of $R$ induces one of $\mathrm{L}^{\perp}(R)$. Here, a representation of an ortholattice $L$ in an inner product space $V$ means an embedding $\eta$ of $L$ into the lattice of all linear subspaces of $V$ such that, for any $u \in L, \eta\left(u^{\perp}\right)$ is the orthogonal of $\eta(u)$. In his thesis [18], the second author established the converse for subdirectly irreducible $R$ (cf. [11]). This involved a coordinatization of representable ortholattices in terms of a variant, including orthogonality conditions, of Jónsson's large partial frames [15]. The purpose of the present note is to give a short presentation to the result, relying on the review of Coordinatization Theory given in [8] and the fact that every variety of $*$-regular rings is generated by its simple members [10].

As general refences we use $[5,1,2]$ for regular and $*$-regular rings, [3] for lattices, $[19,16,20]$ for coordinatization, and [6] for inner product

2000 Mathematics Subject Classification. 06C20; 16E50, 16W10.

Key words and phrases. orthocomplemented modular lattice, *-regular ring, frame, inner prodcut space, representation. 
spaces. Thanks are due to the referee for a lot of helpful suggestions, in particular correcting the proof of the crucial Claims 5.4 and 5.6.

\section{Regular RINGS AND VECTOR SPACE REPRESENTATIONS}

Unless stated otherwise, rings will be associative, with unit 1 as constant (constants in the signature have to be preserved under homomorphisms and in substructures). A (von Neumann) regular ring $R$ is such that for each $a \in R$ there is $x \in R$ such that axa $=a$; equivalently, every right (left) principal ideal is generated by an idempotent.

A representation of a ring $R$ within a vector space $V$ is an embedding of $R$ into the endomorphism $\operatorname{ring} \operatorname{End}(V)$ of $V$. It appears to be well known that every subdirectly irreducible regular ring $R$ admits some representation. Indeed, each maximal left ideal $M_{i}$ of $R$ gives rise to a homorphism $\varphi_{i}: R \rightarrow \operatorname{End}\left(V_{i}\right), \varphi_{i}(r)\left(a+M_{i}\right)=r a+M_{i}$; here $V_{i}$ is the (right) vector space over the division ring of endomorphisms of the simple left $R$-module $R / M_{i}$. These homomorphisms $\varphi_{i}$ yield a subdirect representation of $R$ since $\bigcap_{i} M_{i}=0$ (for $r \neq 0$ and idempotent $e$ with $R r=R e$ choose $M_{i}$ such that $1-e \in M_{i}$ to obtain $\left.r \notin M_{i}=\operatorname{ker} \varphi_{i}\right)$. On the other hand, examples of non-representable regular rings are obtained as products of matrix rings over fields of different characteristics.

We consider lattices $L$ with bounds 0,1 as constants. We use + and $\cap$ to denote joins and meets and write $a \oplus b=c$ if $a+b=c$ and $a \cap b=c$. $L$ is complemented if for any $a$ there is $b$ such that $a \oplus b=1$.

The principal right ideals of a regular ring $R$ form a complemented modular lattice $\mathrm{L}(R)$, a sublattice of the lattice of all right ideals. A representation of a lattice $L$ within a vector space $V$ is an embedding of $L$ into the lattice $\mathrm{L}(V)$ of linear subspaces of $V$. The following is due to Luca Giudici, proof of (1) in [4, Theorem. 4.2.1], cf. [8, Proposition 10.1].

Fact 2.1. If $\iota$ is a representation of the regular ring $R$ in the vector space $V$, then $\eta(a R)=\operatorname{im} \iota(a), a \in R$, defines a representation of $\mathrm{L}(R)$ in $V$.

The purpose of this section is to relate representations the other way round making use of coordinatization results due von Neumann and Jónsson, cf. [8]. A coordinatization of a lattice $L$ is an isomorphism onto $\mathrm{L}(R), R$ a regular ring. Such are based on "frames": suitable coordinate systems. We write $a \sim_{c} b$ if $a+b=a \oplus c=b \oplus c$ and $a \sim b$ if $a \sim_{c} b$ for some $c$. Recall that, for modular $L, a \sim b$ and

$a^{\prime} \leq a$ implies $a^{\prime} \sim b^{\prime}$ for some $b^{\prime} \leq b$. Following Jónsson [15] a large 
partial $n$-frame $\Phi$ of $L$ is given by elements of $L: a_{i}=a_{i i}(0 \leq i<m)$, and $a_{0 i}, 0<i<m$, where $m \geq n$, such that $1=\sum_{i=0}^{m-1} a_{i}, a_{0} \neq 0$, $\sum_{i=0}^{n-1} a_{i}=\bigoplus_{i=0}^{n-1} a_{i}$, and $a_{i} \sim_{a_{0 i}} b_{i}$ for some $b_{i} \leq a_{0}$ for $0<i<m$. Moreover, for $0<i<n$ one requires $b_{i}=a_{0}$. $\Phi$ is a skew $n$-m-frame if, in addition, $1=\bigoplus_{i=0}^{m-1} a_{i}$. Observe that, given such $\Phi, m^{\prime} \leq m, n^{\prime} \leq n$, and $n^{\prime} \leq m^{\prime}$, the $a_{i}, a_{0 i}$ with $i<m^{\prime}$ form a skew $n^{\prime}-m^{\prime}$-frame in the interval $\left[0, \sum_{i=0}^{m^{\prime}-1} a_{i}\right] . \Phi$ is a skew $n$-frame if it is a skew $n$ - $m$-frame for some $m$. From [15, Theorem 1.7] and [8, Proposition 6.2] one obtains the following

Fact 2.2. Every simple complemented modular lattice of height at least $n$ admits some large partial n-frame. Every complemented modular lattice admitting a large partial n-frame also admits a skew n-frame.

In particular this applies to $\mathrm{L}(R), R$ a simple regular ring, due to the following result of Fred Wehrung [21, Theorem 4.3].

Fact 2.3. For a regular ring $R$, the lattice of all congruence relations of $\mathrm{L}(R)$ is ismorphic to the lattice of ideals of $R$.

In presence of a skew $n$-frame, coordinatization, if possible, is unique due to the following result of Jónsson, cf. [8, Theorem 11.2].

Fact 2.4. For regular rings $R, R^{\prime}$, if $\mathrm{L}(R)$ admits a skew $n$-frame, $n \geq$ 3 , then for any isomorphism $\theta: \mathrm{L}(R) \rightarrow \mathrm{L}\left(R^{\prime}\right)$ there is an isomorphism $\iota: R \rightarrow R^{\prime}$ such that $\theta(a R)=\iota(a) R^{\prime}$ for all $a \in R$.

The approach of [8] to coordinatization relied on the following, combining Theorem 7.1 and Corollary 9.2 in [8].

Fact 2.5. For any vector space $V$, complemented sublattice $L$ of $\mathrm{L}(V)$, and skew $n$-frame $\Phi$ in $L, n \geq 3$, there is a regular subring $R_{0}$ of $\operatorname{End}(V)$ and an isomorphism $\omega: \mathrm{L}\left(R_{0}\right) \rightarrow L$ such that $\omega\left(\varphi R_{0}\right)=\operatorname{im} \varphi$ for all $\varphi \in R_{0}$.

Now, we are in position to derive a representation of $R$ from a representation of $\mathrm{L}(R)$.

Proposition 2.6. Given a regular ring $R$, a skew $n$-frame $\Phi, n \geq 3$, in $\mathrm{L}(R)$, a vector space $V$, and an embedding $\eta: \mathrm{L}(R) \rightarrow \mathrm{L}(V)$, there is an embedding $\iota: R \rightarrow \operatorname{End}(V)$ such that $\eta(a R)=\operatorname{im} \iota(a)$ for all $a \in R$.

Proof. Let $L$ denote the sublattice $\eta(\mathrm{L}(R))$ of $\mathrm{L}(V)$. With $R_{0}$ and $\omega$ according to Fact 2.5 one obtains an isomorphism $\omega^{-1} \circ \eta: \mathrm{L}(R) \rightarrow$ $\mathrm{L}\left(R_{0}\right)$. By Fact 2.4 there is an isomorphism $\iota: R \rightarrow R_{0}$ such that $\left(\omega^{-1} \circ\right.$ $\eta)(a R)=\iota(a) R_{0}$ for all $a \in R$. It follows that $\eta(a R)=\omega(\iota(a) R)=$ $\operatorname{im} \iota(a)$ for all $a \in R$. 


\section{3. *-REGULAR RINGS AND INNER PRODUCT SPACES}

A *-ring is a ring $R$ endowed with an involution $r \mapsto r^{*}$. Such $R$ is $*$-regular if it is regular and $r r^{*}=0$ only for $r=0$. A projection is an idempotent $e$ such that $e=e^{*}$; we write $e \in P(R)$. A $*$-ring is $*$-regular if and only if for any $a \in R$ there is $e \in P(R)$ with $a R=e R$; such $e$ is unique. In particular, for $*$-regular $R$, each ideal is closed under the involution. It follows

Fact 3.1. A*-regular ring is simple (subdirectly irreducible) if and only if so is its ring reduct.

For a *-ring $R$ and projection $e \in R$. the corner $e R e$ is the $*$-ring consisting of all eae, $a \in R$, with unit $e$ and operations inherited from $R$, otherwise. The following is Lemma 2 together with Theorem 3 in [10].

Fact 3.2. Given a subdirectly irreducible *-regular ring $R$ with minimal ideal $I$ and a projection $e$ in $I$, the *-ring eRe is *-regular. Moreover, $R$ is a homomorphic image of a *-regular sub-*-ring of an ultraproduct of $*$-rings eRe, where e ranges over the set of projections in $I$.

By an inner product space $V$ we will mean a vector space (also denoted by $V$ ) over a division $*$-ring $F$, endowed with a sesqui-linear form $\langle. \mid$.$\rangle which is anisotropic (\langle v \mid v\rangle=0$ only for $v=0)$ and orthosymmetric $(\langle v \mid w\rangle=0$ if and only if $\langle w \mid v\rangle=0)$. The orthogonal of a subset $X$ is the subspace $X^{\perp}=\{y \in V \mid \forall x \in X .\langle x \mid y\rangle=0\}$. For subspaces $U, W$ of $V$ we write $U \perp W$ if $W \subseteq U^{\perp}$; in this case we write $U+W=U \oplus^{\perp} W$. A subspace $U$ is closed if $U^{\perp \perp}=U$; equivalently, $V=U \oplus^{\perp} W$ for some $W$. Here, $W=U^{\perp}$ and one has the orthogonal projection $\pi_{U}$ where $\pi_{U}(x+y)=x$ for $x \in U$ and $y \in U^{\perp}$. Let End $(V)$ denote the $*$-ring consisting of those endomorphisms $\varphi$ of the vector space $V$ which have an adjoint $\varphi^{*}$ w.r.t. $\langle. \mid$.$\rangle . If \varphi$ is a projection in $\operatorname{End}^{*}(V)$ then $V=\operatorname{im} \varphi \oplus^{\perp} \operatorname{im}\left(\operatorname{id}_{V}-\varphi\right)$. It follows

Fact 3.3. An endomorphism $\varphi$ of $V$ is a projection in $\mathrm{End}^{*}(V)$ if and only if $\varphi=\pi_{U}$, where $U=\operatorname{im} \varphi$.

A representation of a $*$-ring $R$ within $V$ is an embedding of $R$ into End $^{*}(V)$. Of course, any representation $\iota$ of a $*$-ring $R$ within $V$ gives rise to representations of corners $e R e$ within $\operatorname{im} \iota(e)$.

Inner product spaces will be considered as 2-sorted structures with sorts $V$ and $F$. In particular, the class of inner product spaces is closed under formation of ultraproducts. In this setting, representations of *rings $R$ can be viewed as 3-sorted structures (with third sort $R$ ), again forming a class closed under ultraproducts [13, Proposition 13]. On the 
other hand, a representation of $R$ in $V$ gives rise to representations of homomorphic images of $R$ in closed subspaces of certain ultrapowers of $V$ [13, Proposition 25]. It follows

Fact 3.4. In the context of Fact 3.2, if each $*$-ring eRe admits a representation within some inner product space $V_{e}$ for each projection $e \in I$, then the *-ring $R$ admits a representation within a closed subspace of an ultraproduct of spaces $V_{e}$, where e ranges over the set of projections in $I$.

\section{Modular ortholattices}

An ortholattice is a lattice $L$ together with an order reversing involution $a \mapsto a^{\perp}$ such that $1=a \oplus a^{\perp}$. Elements $a, b$ are orthogonal to each other, $a \perp b$, if $b \leq a^{\perp}$; this then implies $a \cap b=0$ and we write $c=a \oplus^{\perp} b$ if $c=a+b$. If $L$ is modular and $u \in L$, then the section $[0, u]$ is again an ortholattice under $a \mapsto u \cap a^{\perp}$; that is, $a, b \leq u$ are orthogonal in $[0, u]$ if and only if they are so in $L$. Also, if $L$ is modular and $a \leq b$ then each of the quotients $b / a,\left(b \cap a^{\perp}\right) / 0$, and $a^{\perp} / b^{\perp}$ generate the same lattice congruence. It follows

Fact 4.1. In a modular ortholattice, any lattice congruence is also a congruence w.r.t. the operation $a \mapsto a^{\perp}$.

The notion of skew frame can be adapted to the ortholattice setting requiring the $a_{i}$ to be pairwise orthogonal, see Niemann [18]. A weaker version will suffice, here. We write $a \sim^{\perp} b$ if $a \perp b$ and $a \sim b$. An orthogonal semiframe in an ortholattice $L$ consists of elements $a_{0}, \ldots, a_{k-1}$ such that $1=\bigoplus_{i=0}^{k-1} a_{i}$ and for each $a_{i}$ there is $b_{i} \sim^{\perp} a_{i}$.

Lemma 4.2. Every modular ortholattice $L$ admitting some skew 2-mframe also admits an orthogonal semiframe. In particular, any simple $L$ of height at least 2 admits an orthogonal semiframe.

Proof. We first observe that the following hold in any modular ortholattice.

(1) If $v \oplus b=1$ and $v^{\perp} \cap b=0$ then $v^{\perp} \sim^{\perp} v^{\prime}$ for some $v^{\prime} \leq v$.

(2) Assume $u \oplus a=1$ and $a \sim^{\perp} a^{\prime}$ for some $a^{\prime} \leq u$. Then there are $d, f$ such that $1=u \oplus^{\perp} d \oplus^{\perp} f, d \sim^{\perp} d^{\prime}$ and $e \sim^{\perp} e^{\prime}$ for some $d^{\prime} \leq u$ and $e^{\prime} \leq u+d$.

(1) follows from $v^{\perp} \sim_{b} v \cap\left(v^{\perp}+b\right)$. To prove (2), put $d:=a \cap u^{\perp}$ and $v:=u+d$. Then $v=u \oplus^{\perp} d$ and $d \sim^{\perp} d^{\prime}$ for some $d^{\prime} \leq a^{\prime} \leq u$. Moreover, $a \cap v^{\perp}=a \cap d^{\perp} \cap u^{\perp}=d \cap d^{\perp}=0$. Now, put $b:=a \cap d^{\perp}$, the orthocomplement of $d$ in the ortholattice [0,a]; thus, $b \oplus d=a$ and 
$v \oplus b=1$. On the other hand, from $b \leq a$ it follows $b \cap v^{\perp}=0$. Now, $1=u \oplus d \oplus v^{\perp}$ and (2) follows applying (1).

Finally, observe that (2) deals with the case $m=2$ as well with the inductive step from $m-1$ to $m$. The second claim follows from Facts 2.2 and 4.1

A representation of an ortholattice $L$ in an inner product space $V$ is an embedding $\eta$ of the lattice $L$ into $\mathrm{L}(V)$ such that $\eta\left(a^{\perp}\right)=\eta(a)^{\perp}$ for all $a \in L$. In particular, $\eta(L)$ is a modular sub-ortholattice of the (in general, non-modular) lattice of all closed subspaces of $V$.

Fact 4.3. Given a *-regular ring $R$, the lattice $\mathrm{L}(R)$ expands to an ortholattice $\mathrm{L}^{\perp}(R)$ defining $(a R)^{\perp}=(1-e) R$ where $e \in P(R)$ such that $a R=e R$. In particular, for $e, f \in P(R)$ one has $e R \subseteq f R$ if and only if $f e=e$.

For $e, f \in P(R)$, we write $e \perp f$ if $e R \perp f R$; that is, $f e=0=e f$. Now, in view of Fact 3.3, Fact 2.1 transfers as follows.

Fact 4.4. If $\iota$ is a representation of the $*$-regular ring $R$ in the inner product space $V$ then $\eta(a R)=\operatorname{im} \iota(a)$, defines a representation of the ortholattice $\mathrm{L}^{\perp}(R)$ in $V$.

In the presence of orthogonal semiframes, we will relate such representations the other way round.

\section{MAIN LEMMA}

Lemma 5.1. Given a *-regular ring $R$, an orthogonal semiframe $\Phi$ in $\mathrm{L}^{\perp}(R)$, an inner product space $V$, and representation $\iota$ of the ring $R$ in the vector space $V$, then $\iota$ is a representation of the $*$-ring $R$ within $V$, provided that $\eta: \mathrm{L}^{\perp}(R) \rightarrow \mathrm{L}(V), \eta(a R):=\operatorname{im} \iota(a), a \in R$, defines an ortholattice representation in the inner product space $V$.

Recall that $\eta(a R)=\eta(b R)$ if $a R=b R$ (and we may write $\eta(a):=$ $\eta(a R))$ and that $\eta$ is a lattice representation in view of Fact 2.1. Thus, the point is to show $\iota\left(a^{*}\right)=\iota(a)^{*}$ for all $a \in R$ using the fact that $\eta$ preserves orthogonality. For the remainder of this section we assume the hypotheses of the Lemma.

Claim 5.2. Consider closed subspaces $U, W$ of $V$ such that $U \perp W$ and $\varphi, \psi \in \operatorname{End}(V)$ such that $\varphi=\pi_{W} \varphi \pi_{U}$ and $\psi=\pi_{U} \psi \pi_{W}$. Then $\psi=\varphi^{*}$ if and only if $\operatorname{im}\left(\pi_{U}-\varphi\right) \perp \operatorname{im}\left(\pi_{W}+\psi\right)$.

Proof. This follows immediately since for all $v, w \in V$ one has

$$
\left\langle\left(\pi_{U}-\varphi\right)(v) \mid\left(\pi_{W}+\psi\right)(w)\right\rangle=\left\langle\pi_{U}(v) \mid \psi(w)\right\rangle-\left\langle\varphi(v) \mid \pi_{W}(w)\right\rangle
$$


Claim 5.3. If $e \perp f$ in $P(R)$ and $a \in f$ Re then $\iota\left(a^{*}\right)=\iota(a)^{*}$.

Proof. Put $b=a^{*}$. Then $b \in e R f$ and $(e-a)^{*}(f+b)=0$, that is $(e-a) R \perp(f+b) R$. It follows $\eta(e-a) \perp \eta(f+b)$. Now, $\eta(e-a)=$ $\operatorname{im} \iota(e-a)=\operatorname{im}(\iota(e)-\iota(a))$ and $\eta(f+b)=\operatorname{im}(\iota(f)+\iota(b))$ and Claim 5.2 applies with $\varphi=\iota(a), U=\operatorname{im} \iota(e), \psi=\iota(b), W=\operatorname{im} \iota(f)$.

Claim 5.4. If $e R \sim f R$ in $\mathrm{L}^{\perp}(R)$ for idempotents e, $f \in R$ then there is $c \in f$ Re such that $c x=c y$ implies $x=y$ for all $x, y \in e R e$.

Proof. Assume $e R \sim_{g R} f R$; then $\omega(x)=y \Leftrightarrow x-y \in g R$ defines an isomorphism $\omega: e R \rightarrow f R$ of right $R$-modules. Put $c=\omega(e) e$ and observe that $\omega(x)=\beta(e x)=\beta(e) x=c x$ for all $x \in e$ Re. Thus, assuming $c x=c y$ for given $x, y \in e R e$ it follows $\omega(x)=c x=c y=\omega(y)$ whence $x=y$.

Claim 5.5. Consider closed subspaces $U \perp W$ of $V$ and $\varepsilon \in \operatorname{End}^{*}(V)$ such that $\varepsilon \circ \xi=\varepsilon \circ \chi$ implies $\xi=\chi$ for all $\xi, \chi \in \pi_{U} \circ \mathrm{End}^{*}(V) \circ \pi_{U}$. Then $\varphi^{*}=\psi$ provided that $\varphi, \psi \in \pi_{U} \circ \operatorname{End}^{*}(V) \circ \pi_{U}$ and $(\varepsilon \circ \varphi)^{*}=\psi \circ \varepsilon^{*}$.

Proof. From $\varphi^{*} \circ \varepsilon^{*}=(\varepsilon \circ \varphi)^{*}=\psi \circ \varepsilon^{*}$ it follows $\varepsilon \circ \varphi=\varepsilon \circ \psi^{*}$, whence $\varphi=\psi^{*}$ and $\varphi^{*}=\psi$.

Claim 5.6. Given $e, f$ as in Claim 5.4 such that $e \perp f$ one has $\iota\left(a^{*}\right)=$ $\iota(a)^{*}$ for all $a \in e R e$.

Proof. We put $b=a^{*}$ and have to show $\iota(b)=\iota(a)^{*}$. Choose $c$ according to Claim 5.4. By Claim 5.3 one has $\iota\left(c^{*}\right)=\iota(c)^{*}$ and $\iota\left((c a)^{*}\right)=(\iota(c a))^{*}$ since $c, c a \in f R e$. It follows $\iota(b) \iota(c)^{*}=\iota(b) \iota\left(c^{*}\right)=$ $\iota\left(b c^{*}\right)=\iota\left((c a)^{*}\right)=(\iota(c a))^{*}=(\iota(c) \iota(a))^{*}$ whence $\iota(b)=\iota(a)^{*}$ applying Claim 5.5 with $U=\operatorname{im} e, W=\operatorname{im} f, \varphi=\iota(a), \psi=\iota(b)$, and $\varepsilon=\iota(c)$.

Proof. of the Lemma. We fix an orthogonal semiframe $\Phi$ of $\mathrm{L}^{\perp}(R)$, that is pairwise orthogonal projections $e_{i}, 0 \leq i<k$, such that $\bigoplus_{i=0}^{k-1} e_{i} R=$ $R$ and for each $i<k$ there are $f_{i}, g_{i} \in P(R)$ with $e_{i} R \sim^{\perp} f_{i} R$. By Claims 5.3 and 5.6 one has $\iota\left(a^{*}\right)=\iota(a)^{*}$ for all $a \in e_{j} R e_{i}, i, j<k$.

Now, $e_{i} e_{j}=0$ for $i \neq j$ since $e_{i} \perp e_{j}$. Thus $e=\sum_{i 0}^{k-1} e_{i}$ is a projection and $e R=R$ whence $e=1$ by uniqueness. It follows, for each $a \in R$, that $a=\sum_{i, j=0}^{k-1} e_{j} a e_{i}$ and $a^{*}=\sum_{i, j=0}^{k-1} e_{j} a^{*} e_{i}$. Thus $\iota(a)^{*}=\left(\sum_{i, j=0}^{k-1} \iota\left(e_{j} a e_{i}\right)\right)^{*}=\sum_{i, j=0}^{k-1}\left(\iota\left(e_{j} a e_{i}\right)\right)^{*}=\sum_{i, j=0}^{k-1} \iota\left(\left(e_{j} a e_{i}\right)^{*}\right)=$ $\iota\left(\sum_{i, j=0}^{k-1}\left(e_{j} a e_{i}\right)^{*}\right)=\iota\left(\sum_{i, j=0}^{k-1} e_{i} a^{*} e_{j}\right)=\iota\left(a^{*}\right)$.

\section{Results}

Facts 3.1, 2.3, and 2.2 yield the following. 
Fact 6.1. A simple *-regular ring admits a large partial $n$-frame if $\mathrm{L}(R)$ is of height at least $n$.

Theorem 6.2. Given a *-regular ring $R$ such that its ortholattice $\mathrm{L}^{\perp}(R)$ of projections admits a large partial $n$-frame, $n \geq 3$, and a representation $\eta$ within some inner product space $V$. Then there is a representation $\iota$ of the $*$-ring $R$ within $V$ such that $\eta(a)=\operatorname{im} \iota(a)$ for all $a \in R$.

Proof. By Fact 2.2 one has a skew $n$-frame, $n \geq 3$ and so Proposition 2.6 provides the ring embedding $\iota: R \rightarrow \operatorname{End}(V)$ such that $\eta(a)=\operatorname{im} \iota(a)$. Now, by Lemma 4.2 there is an orthogonal semiframe and Lemma 5.1 shows that $\iota$ is a representation of the $*$-ring $R$.

Corollary 6.3. Consider a subdirectly irreducible $*$-regular ring $R$ such that $\mathrm{L}^{\perp}(R)$ is of height at least 3 and has a representation in the inner product space $V$. Then the $*$-ring $R$ has a representation within a closed subspace of some ultrapower of $V$.

Proof. Let $P$ denote the set of projections $e$ in the minimal ideal $I$ of $R$ such that $\mathrm{L}^{\perp}(e R e)$ has height at least 3. Observe that for any projection $f \in I, f R f$ embeds into $e R e$ for some $e \in P$. Also, for $e \in P, \mathrm{~L}^{\perp}(e R e)$ is a section of $\mathrm{L}^{\perp}(R)$ and any representation $\eta$ of $\mathrm{L}^{\perp}(R)$ in some inner product space $V$ restricts to a representation of $\mathrm{L}^{\perp}(e R e)$ in a closed subspace $V_{e}$ of $V$. By Lemma 5.1 one obtains a representation of the $*$-ring $e R e$ within $V_{e}$ for each $e \in P$. By Fact 3.4 this gives rise to a representation of $R$ in an ultraproduct of the $V_{e}$, that is a closed subspace of an ultrapower of $V$.

Let $\mathcal{M O} \mathcal{L}$ and $\mathcal{M O} \mathcal{L}_{\text {art }}$ denote the ortholattice varieties generated by all respectively all finite height modular ortholattices.

Corollary 6.4. A *-regular ring $R$ is a subdirect product of representables if and only if $\mathrm{L}^{\perp}(R) \in \mathcal{M O} \mathcal{L}_{\text {art }}$.

Proof. Consider a homomorphism $\iota_{k}$ of $R$ onto $S_{k}$. Then $S_{k}$ is also *-regular and $\iota_{k}$ induces a homomorphism $\eta_{k}$ of $\mathrm{L}^{\perp}(R)$ onto $\mathrm{L}^{\perp}\left(S_{k}\right)$ given by $\eta_{k}(e R)=\iota_{k}(e) S_{k}$, see Proposition 5.4(iv) [14]. Moreover, the $\iota_{k}$ yield a subdirect decomposition if and only if so do the $\eta_{k}$.

Thus, it suffices to consider subdirectly irreducible $R$; that is, subdirectly irreducible $\mathrm{L}^{\perp}(R)$. If $R$ is representable then, by Fact 2.3, $\mathrm{L}^{\perp}(R)$ is representable, too, and so in the variety generated by subspace ortholattices of finite dimensional inner product spaces by $[14$, Theorem 10.1], whence in $\mathcal{M O} \mathcal{L}_{\text {art }}$. For the converse, we may assume that $\mathrm{L}^{\perp}(R)$ is of height at least 4 , since otherwise $R$ is simple artinian 
whence representable. Now any finite height modular ortholattice is a direct product of simple ones and, by Jónsson's Lemma, $\mathrm{L}^{\perp}(R)$ is is a homomorphic image of a sub-ortholattice of an ultraproduct of such $L_{i}$. Since $\mathrm{L}^{\perp}(R)$ contains a 5 -element chain, the ultraproduct may be restricted to be formed from the $L_{i}$ of height at least 4 . Such are representable whence, by Lemma 8.3 and Corollary 8.6 in [14], so is $\mathrm{L}^{\perp}(R)$. The claim follows by Corollary 6.3.

Corollary 6.5. $\mathcal{M O} \mathcal{L}=\mathcal{M O} \mathcal{L}_{\text {art }}$ if and only if every subdirectly irreducible $*$-regular ring is representable.

Proof. Assume that every subdirectly irreducible $*$-regular ring is representable. According to [12] it suffices to show $L \in \mathcal{M O} \mathcal{L}_{\text {art }}$ for each simple $L \in \mathcal{M O} \mathcal{L}$. Of course, we have to consider $L$ of infinite height, only. Such $L$ is coordinatizable, that is $L \cong \mathrm{L}^{\perp}(R)$ for some $*$-regular ring $R$ (cf. [17, Section 4.3] respectively Fact 2.2 and Theorems 11.2 and 13.2 in [8]). Thus, by Fact $2.3, L$ is representable, whence in $\mathcal{M O} \mathcal{L}_{\text {art }}$ cf. [14, Theorem 10.1]. The other direction is immediate by Corollary 6.4.

In case of *-regular rings $R$ without unit, $R$ is the directed union of the $e R e, e$ a projection in $R$, and $\mathrm{L}^{\perp}(R)$ the directed union of the $\mathrm{L}^{\perp}(e R e)$. The latter is a modular sectional ortholattice $L$, a modular lattice with 0 and a binary operation $(a, u) \mapsto a^{\perp_{u}}$ such that $a \mapsto a^{\perp_{u}}$ is an orthocomplementation on $[0, u]$ and $a^{\perp_{v}}=a^{\perp_{u}} \cap v$ if $v \leq u$. A representation of such $L$ is given by an inner product space $V$ and an embedding $\eta$ of the lattice $L$ with 0 into $\mathrm{L}(V)$ such that, for each $u \in L$, $\eta(u)$ is closed in $V$ and the restriction of $\eta$ is a representation of the ortholattice $[0, u]$ within $\eta(u)$.

Corollary 6.6. Corollaries 6.3 and 6.4 hold for $*$-regular rings without unit, analogously.

\section{REFERENCES}

[1] S. K. Berberian, Baer *-rings, Springer, Grundlehren 195, Berlin 1972.

[2] S. K. Berberian, Baer rings and Baer *-rings, Austin, 1988, http://www . ma. utexas.edu/mp \_arc/c/03/03-181.pdf.

[3] P. Crawley and R. P. Dilworth, Algebraic Theory of Lattices, Prentice-Hall, Englewood Cliffs, 1973.

[4] L. Giudici, Dintorni del Teorema di Coordinatizatione di von Neumann, Ph.D. thesis, Univ. di Milano, 1995, http://www. nohay.net/mat/tesi.1995/tesi.ps . gz.

[5] K. R. Goodearl, Von Neumann Regular Rings, second edition, Krieger, Malabar, 1991. 
[6] H. Gross, Quadratic Forms in Infinite Dimensional Vector Spaces, Birkhäuser, Basel, 1979.

[7] D. Handelman, Finite Rickart $C^{*}$-algebras and their properties, in Studies in Analysis, ed. G.-C. Rota, pp. 171-196, Adv. in Math. Suppl. Stud., 4, Academic Press, New York-London, 1979.

[8] C. Herrmann, A review of some of Bjarni Jnsson's results on representation of Arguesian lattices, Algebra Universalis, 70 (2013), 163-174.

[9] C.Herrmann, Direct finiteness of representable regular *-rings, Algebra Universalis, 80 (2019), no. 1, Art. 3, 5 pp. https://arxiv.org/abs/1904.04505.

[10] C. Herrmann, Varieties of *-regular rings, Math. Slovaca (to appear), https: //arxiv.org/abs/1904.04505.

[11] C.Herrmann and N.Niemann, On linear representation of $*$-regular rings having representable ortholattice of projections, https:arXiv:1811.01392.

[12] C. Herrmann and M.S. Roddy, A second note on the equational theory of modular ortholattices, Algebra Universalis, 57 (2007), 371-373.

[13] C. Herrmann and M.V. Semenova, Rings of quotients of finite AW-algebras. Representation and algebraic approximation (Russian), Algebra Logika, 53 (2014), 466-504; translation in Algebra Logic, 53 (2014), 298-322.

[14] C. Herrmann and M.V. Semenova, Linear representations of regular rings and complemented modular lattices with involution, Acta Sci. Math. (Szeged), 82 (2016), 395-442.

[15] B. Jónsson, Representations of complemented modular lattices, Trans. Amer. Math. Soc.. 97 (1960), 64-94.

[16] F. Maeda, Kontinuierliche Geometrien, Springer, Berlin, 1958.

[17] F. MicOL, On representability of *-regular rings and modular ortholattices, Ph.D. thesis, TU Darmstadt, January 2003, http://elib.tu-darmstadt.de/ diss/000303/diss.pdf.

[18] N. NiEMANN, On representability of *-regular rings and regular involutive rings in endomorphism rings of vector spaces, Ph.D. thesis, TU Darmstadt 2007, Logos Verlag Berlin 2007.

[19] J. von Neumann, Continuous Geometries, Princeton Univ. Press, Princeton 1960.

[20] L. A. Skornyakov, Complemented Modular Lattices and Regular Rings, Oliver \& Boyd Edinburgh 1964.

[21] F. Wehrung, A uniform refinement property for congruence lattices, Proc. Amer. Math. Soc., 127 (1999), 363-370.

(C. Herrmann) TUD FB4, Schlossgartenstr. 7, 64289 Darmstadt, GerMANY

E-mail address: herrmann@mathematik.tu-darmstadt.de 